\title{
Superior Methanol Electrooxidation Performance of (110)-Faceted Nickel Polyhedral Nanocrystals
}

Junshan Li, ${ }^{\mathrm{a}, \mathrm{b}}$ Yong Zuo, ${ }^{\mathrm{a}, \mathrm{b}}$ Junfeng Liu, ${ }^{\mathrm{a}, \mathrm{b}}$ Xiaoting Yu, ${ }^{\mathrm{a}, \mathrm{b}}$ Ruifeng Du, ${ }^{\mathrm{a}, \mathrm{b}}$ Ting Zhang, ${ }^{\mathrm{c}}$ Maria F. InfanteCarrió, ${ }^{\mathrm{c}}$ Pengyi Tang, ${ }^{\mathrm{c}}$ Jordi Arbiol, ${ }^{\mathrm{c}, \mathrm{d}}$ Jordi Llorca, ${ }^{\mathrm{e}}$ Zhishan Luo, ${ }^{\mathrm{a}^{*}}$ Andreu Cabot ${ }^{\mathrm{a}, \mathrm{d}, *}$

a Catalonia Institute for Energy Research - IREC, Sant Adrià de Besòs, Barcelona, 08930, Spain

$b$ Department of Electronics and Biomedical Engineering, Universitat de Barcelona, 08028 Barcelona, Spain

c Catalan Institute of Nanoscience and Nanotechnology (ICN2), CSIC and BIST, Campus UAB, Bellaterra, 08193 Barcelona, Spain

d ICREA, Pg. Lluís Companys 23, 08010 Barcelona, Spain

e Institute of Energy Technologies, Department of Chemical Engineering and Barcelona Research Center in Multiscale Science and Engineering. Universitat Politècnica de Catalunya, EEBE, 08019 Barcelona, Spain

\begin{abstract}
We present the synthesis of (110) faceted nickel polyhedral nanocrystals (NCs) and their characterization as electrocatalysts for the methanol oxidation reaction (MOR). Faceted Ni NCs were produced at $180{ }^{\circ} \mathrm{C}$ through the reduction in solution of a Ni salt with $\mathrm{TBAB}$ in the presence of proper surfactants. They were combined with carbon black and Nafion and deposited over glassy carbon to study their electrocatalytic properties. (110) Ni NCs displayed a first order reaction with $\mathrm{KOH}$ in the concentration range from $0.1 \mathrm{M}$ to 1.0 M. A higher coverage of active species, but lower rate limiting diffusion coefficients were calculated when comparing with electrodes prepared from spherical Ni NCs. Overall, electrodes based on faceted $\mathrm{Ni}$ NCs displayed excellent performance at $0.6 \mathrm{~V} v s . \mathrm{Hg} / \mathrm{HgO}$ in $1.0 \mathrm{M} \mathrm{KOH}$ containing $1.0 \mathrm{M}$ methanol with very high current densities, up to $61 \mathrm{~mA} \mathrm{~cm}^{-2}$ and unprecedented mass activities up to $2016 \mathrm{~mA} \mathrm{mg}^{-1}$. Electrodes based on (110) faceted Ni NCs also displayed a notable stability. While they suffered an initial activity loss of ca. 30\% during the first $10000 \mathrm{~s}$ operation, activity stabilized at very high current densities, $35 \mathrm{~mA} \mathrm{~cm}^{-2}$, and mass activities, $\sim 1200 \mathrm{~mA} \mathrm{mg}^{-1}$, with only a $0.5 \%$ decrease during operation from 20000 to $30000 \mathrm{~s}$.
\end{abstract}

Keywords: Nanocrystals; nickel; electrocatalyst; methanol oxidation reaction; direct methanol fuel cell. 


\section{INTRODUCTION}

Direct methanol fuel cells (DMFCs) are characterized by low operating temperatures, a relatively simple architecture, high energy efficiencies, a high fuel energy density and convenient fuel storage and transportation. ${ }^{1-4}$ In spite of their advantages over competing fuel cell technologies, the requirement of Ptbased electrocatalysts restricts their commercialization to niche markets where cost-efficiency limitations are not a major concern. ${ }^{5,6}$

The widespread implementation of DMFCs requires the development of cost-effective electrocatalysts for the methanol oxidation reaction (MOR). In this direction, $\mathrm{Ni}$ is considered a main alternative to noble metals, exhibiting excellent performance towards MOR in alkaline media. ${ }^{7-9}$ To optimize its performance, Ni has been alloyed with different elements and it has been combined or grown on a variety of conductive supports. ${ }^{10-12}$ Additionally, its dimensions have been reduced to the nanometer scale to maximize surface area and thus density of surface site. ${ }^{13,14}$ Another important parameter that strongly influence performance is the NC surface chemistry and exposed facets. ${ }^{15-17}$ In related and more studied Pt catalyst, single crystal $\mathrm{Pt}(110)$ electrodes with more open atomic structures exhibit the highest electrocatalytic activities. ${ }^{18-21}$ However, (110) facets of single crystals are also considered to be more prone to be poisoned and thus to provide lower stabilities. We hypothesize that using highly faceted NPs, we can take advantage of the highest performance of the most active facet, while at the same time, the large density of interfaces and vertices in small nanoparticles can provide active sites to reduce poisoning.

Aiming at taking advantage of high activity facets, polyhedral Ni NCs with predominant (110) facets were produced in solution through the use of proper organic ligands. After removing these insulating ligands, $\mathrm{Ni}$ NCs were mixed with carbon black and Nafion and deposited on a glassy carbon support. The performance of such electrocatalysts toward MOR was subsequently analyzed by means of cyclic voltammetry (CV) and chronoamperometry (CA). 


\section{EXPERIMENTAL}

Chemicals: $\mathrm{Nickel(II)}$ acetylacetonate $\left(\mathrm{Ni}(\mathrm{acac})_{2} \cdot \mathrm{xH}_{2} \mathrm{O}(\mathrm{x} \sim 2)\right.$, 95\%, Sigma-Aldrich), tri-n-octylphosphine (TOP, 97\%, Strem), oleylamine (OAm, 80-90\%, TCI), borane tert-butylamine complex (TBAB, 97\%, Sigma-Aldrich), oleic acid (OAc, Sigma-Aldrich), Nafion (10 wt. \%, perfluorinated ion-exchange resin, dispersion in water), methanol (anhydrous, 99.8\%, Sigma-Aldrich), carbon black (CB, Vulcan XC72), potassium hydroxide $(\mathrm{KOH}, 85 \%$, Sigma-Aldrich), trioctylphosphine oxide (TOPO, 99\%, Sigma-Aldrich) and ammonium thiocyanate $\left(\mathrm{NH}_{4} \mathrm{SCN}\right.$, ACS reagent, $\geq 97.5 \%$, Sigma-Aldrich) were used as received without further purification. Hexane, acetone and ethanol were of analytical grade and purchased from various sources. An argon-filled glove-box was used for storing and dealing with sensitive chemicals.

Ni polyhedral NCs: All syntheses were conducted using standard airless techniques. To prepare Ni polyhedral NCs, a solution of $10 \mathrm{~mL} \mathrm{OAm}$ and $0.5 \mathrm{~mL} \mathrm{OAc}$ together with $0.4 \mathrm{mmol} \mathrm{Ni(acac})_{2} \cdot \mathrm{xH}_{2} \mathrm{O}$ were loaded into a $25 \mathrm{~mL}$ three-necked flask connected a vacuum/dry argon gas Schlenk line and containing a magnetic bar. The reaction was strongly stirred and degassed under vacuum at $80{ }^{\circ} \mathrm{C}$ for 1 hour to remove water, air, and other low-boiling point impurities. Then, a gentle flow of argon was introduced. Meanwhile, $0.5 \mathrm{mmol}$ TBAB was dissolved in $0.5 \mathrm{~mL}$ OAm and them the mixture was sonicated for half an hour and degassed for an additional hour. Subsequently, the reaction flask was heated to $180{ }^{\circ} \mathrm{C}$ within a ramp of $5{ }^{\circ} \mathrm{C} / \mathrm{min}$. At this temperature, the prepared reductant mixture was injected. Upon injection, a visible color change, from deep green to black was immediately observed. The reaction was maintained at this temperature for 1 hour, followed by a rapid cool down to room temperature using a water bath. The content of the reaction mixture were centrifuged at $6000 \mathrm{rpm}$ for $3 \mathrm{~min}$ with help of acetone as polar solvent. The isolated powder was suspended using hexane and acetone and then centrifuged again. This entire process was repeated twice. Finally, the NCs were suspended in $5 \mathrm{~mL}$ hexane in a vial for further use.

Ni spherical NCs: $13 \mathrm{~nm} f c c$-Ni NCs were prepared following the procedure described by Y. Chen et al. ${ }^{22} \mathrm{In}$ a typical synthesis, $1 \mathrm{mmol} \mathrm{Ni(acac})_{2}, 7 \mathrm{ml} \mathrm{OAm}, 0.4 \mathrm{mmol}$ TOP and $0.25 \mathrm{mmol}$ TOPO were loaded in a three-neck flask and stirred under a gentle flow of argon. Temperature was raised to $130{ }^{\circ} \mathrm{C}$ and kept for 20 min. Then, the solution was quickly heated to $215{ }^{\circ} \mathrm{C}$ and maintained at this temperature for $45 \mathrm{~min}$. Subsequently, the flask was cooled down to room temperature using a water bath. The black precipitate was separated through centrifugation after adding ethanol. NCs were re-dispersed and precipitated three times using hexane and ethanol as solvent and non-solvent, respectively. The product was finally dispersed in hexane.

Characterization: Powder X-ray diffraction (XRD) was measured on a Bruker AXS D8 Advance X-ray diffractometer with $\mathrm{Cu} \mathrm{K}$ radiation $(\lambda=1.5106 \AA)$ operating at $40 \mathrm{kV}$ and $40 \mathrm{~mA}$. Scanning electron microscopy (SEM) analyses were performed on a ZEISS Auriga SEM with an energy dispersive X-ray spectroscopy (EDS) detector at $20 \mathrm{kV}$. Transmission electron microscopy (TEM) analyses were carried out 
on a ZEISS LIBRA 120, operating at $120 \mathrm{kV}$, using a 200 mesh Carbon-coated grid from Ted-Pella as substrate. High-resolution TEM (HRTEM) and scanning TEM (STEM) studies were carried out using a field emission gun FEI Tecnai F20 microscope at $200 \mathrm{kV}$ with a point-to-point resolution of $0.19 \mathrm{~nm}$. High angle annular dark-field (HAADF) STEM was combined with electron energy loss spectroscopy (EELS) in the Tecnai microscope by using a GATAN QUANTUM filter. X-ray photoelectron spectroscopy (XPS) analyses were performed on a SPECS system equipped with an Al anode XR50 source operating at $150 \mathrm{~mW}$ and a Phoibos 150 MCD-9 detector. The pressure in the analysis chamber was kept below $10^{-7} \mathrm{~Pa}$. The area analyzed was about $2 \mathrm{~mm} \times 2 \mathrm{~mm}$. The pass energy of the hemispherical analyzer was set at $25 \mathrm{eV}$ and the energy step was maintained at $0.1 \mathrm{eV}$. Data processing was performed with the Casa XPS program (Casa Software Ltd., UK). Binding energies were shifted according to the reference $\mathrm{C}$ 1s peak that was located at $284.8 \mathrm{eV}$. Fourier transformed infrared (FTIR) spectroscopy data were recorded on an Alpha Bruker spectrometer before and after ligand exchange.

Ligand exchange: Native organic ligands were displaced from the $\mathrm{NC}$ surface using a $\mathrm{NH}_{4} \mathrm{SCN}$ solution according to previously published reports. ${ }^{23}$ In a typical procedure, $5 \mathrm{~mL}$ of a $0.13 \mathrm{M}\left(1.0 \mathrm{~g} \mathrm{NH}_{4} \mathrm{SCN}\right.$ in 100 $\mathrm{mL}$ acetone) $\mathrm{NH}_{4} \mathrm{SCN}$ solution in acetone was added to $5 \mathrm{~mL}$ as-synthesized NCs in hexanes and the resulting solution agitated for $2 \mathrm{~min}$ and maintained unperturbed for another $10 \mathrm{~min}$. The, the solution was centrifuged at low speed and the supernatant was discarded. Further washing process was repeated by centrifuging with equal volume of acetone and hexane. Finally, the precipitated NCs were dried under vacuum overnight.

Electrochemical characterization: Electrochemical measurements were conducted at room temperature on AutoLab and Metrohm workstations using conventional three-electrode systems: a counter electrode (Pt mesh), a working electrode (glassy carbon) and a reference electrode $(\mathrm{Hg} / \mathrm{HgO})$. The catalytic ink was prepared by mixing $2 \mathrm{mg}$ of NCs together with $4 \mathrm{mg}$ of CB in $1.6 \mathrm{~mL}$ MilliQ water/ethanol $(\mathrm{v} / \mathrm{v}=1: 1)$ and $100 \mu \mathrm{L}$ of a $10 \mathrm{wt} \%$ Nafion solution. The working electrode was prepared by a drop-casting method. Specifically, $5 \mu \mathrm{L}$ ink was pipetted onto the polished and carefully washed glassy carbon electrode, and then it was allowed to dry naturally in open air. The $\mathrm{Hg} / \mathrm{HgO}$ reference electrode was placed in a salt bridge of 1.0 $\mathrm{M} \mathrm{KOH}$. Prior to each experiment, the alkaline electrolyte was purged with high-purity $\mathrm{N}_{2}$ gas for $30 \mathrm{~min}$. All potential values presented in this paper were referred to the reference electrode, vs. $\mathrm{Hg} / \mathrm{HgO}$. Cyclic voltammetry $(\mathrm{CV})$ and chronoamperometry $(\mathrm{CA})$ measurements were performed to investigate the activity and stability for MOR. The current densities were modified by the geometric surface area of the GC electrode $\left(0.196 \mathrm{~cm}^{-2}\right)$ or the metal mass loading $(\sim 5.9 \mu \mathrm{g} \mathrm{NCs})$. 


\section{RESULTS AND DISCUSSION}

Ni NCs were produced at $180{ }^{\circ} \mathrm{C}$ from the reaction of nickel acetylacetonate with $\mathrm{TBAB}$ in the presence of OAm and OAc (see experimental section for details). Figure 1a shows a representative TEM micrograph of the produced material. Ni NCs displayed a highly faceted polyhedral geometry and had an average size of 16 $\pm 2 \mathrm{~nm}$, as shown in Figure 1b. XRD analysis showed the Ni NCs to have the $f c c$ crystal phase and displayed no peaks corresponding to any additional crystalline secondary phase (Figure 1c).
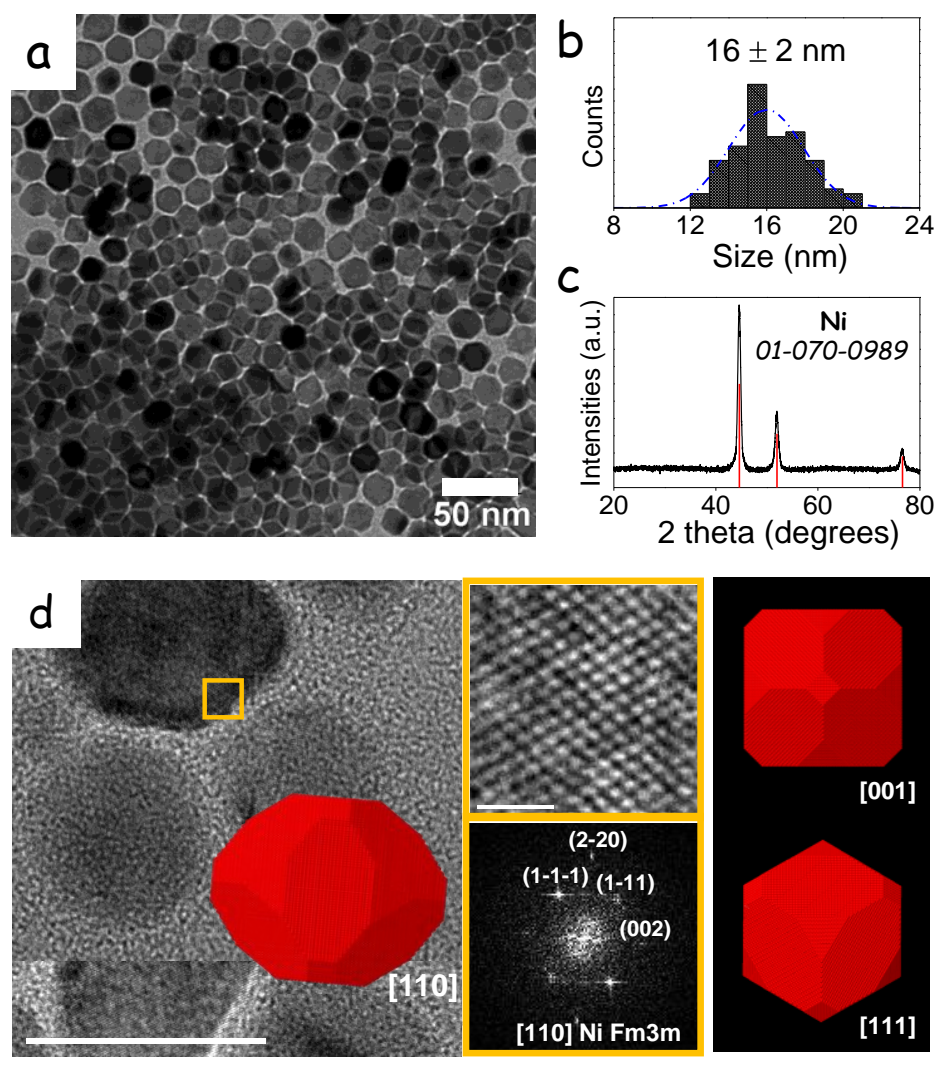

Figure 1. (a) Representative TEM micrograph of Ni polyhedral NCs. (b) Size distribution histogram. (c) XRD pattern of Ni NCs including the JCPDS 01-070-0989 reference corresponding to the Ni fcc-phase. (d) HRTEM micrograph of Ni NCs, detail of the orange squared region and its corresponding indexed power spectrum, and 3D atomic models of a $\mathrm{NC}$ visualized along two different orientations as noted and displaying a multifaceted morphology with predominant (110) facets. The inset of the HRTEM micrograph corresponds to a $3 \mathrm{D}$ atomic model of the selected $\mathrm{NC}$, which is oriented to be visualized from its [110] zone axis.

Extensive HRTEM characterization confirmed the Ni NCs to display a cubic crystal phase (space group = Fm3m) with $\mathrm{a}=\mathrm{b}=\mathrm{c}=3.5157 \AA$ (Figure 1d). ADF-STEM characterization and EELS chemical composition maps showed the Ni NCs to have a homogeneous composition, although a thin oxygen-rich shell could be discerned on its surface (Figure S1). This shell was related both to the presence of oxygen and oxygen-containing species bond to the NC surface and to a slight oxidation of the material during manipulation and transportation before TEM analysis. 
XPS analysis showed that the surface of Ni NCs that had been exposed to the ambient atmosphere presented two different chemical phases of $\mathrm{Ni}: \mathrm{Ni}^{0}$ and at least one oxidized $\mathrm{Ni}$ phase which accounted for close to $60 \%$ of the Ni (Figure S2). Taking into account the reduced thickness probed by XPS, we estimate the oxidation layer on the surface of the air-exposed NCs to extend around 1-2 nm. ${ }^{24,25}$

While the formation of these faceted Ni NCs relied on the use of organic ligands to adjust surface energy and provide suitable crystal growth environment, the presence of these organic molecules could strongly limit their electrical properties and ability to interact with the media. Therefore, before their application as electrocatalysts, organic ligands were removed using a $0.13 \mathrm{M} \mathrm{NH}_{4} \mathrm{SCN}$ solution (see experimental section for details). ${ }^{23}$ After ligand exchange process, samples could not be re-dispersed in organic solvents such as hexane, and IR absorption bands corresponding to the $\mathrm{C}-\mathrm{H}$ vibration modes $\left(2851-2923 \mathrm{~cm}^{-1}\right)$ completely disappeared from the FTIR spectrum (Figure S3), proving its effective removal. ${ }^{26}$

Subsequently, NCs were mixed with carbon black and Nafion in a mixture of water/isopropanol (1:1). The obtained ink was drop-casted on a glassy carbon electrode (see experimental section for details) to produce the electrocatalysts to be tested toward MOR. For the sake of comparison with non-faceted particles, $13 \mathrm{~nm}$ spherical Ni NCs were prepared according to previously published works (see experimental section and Figures S4-5) and treated in the same manner as the Ni polyhedrons to produce the corresponding electrocatalysts.

The electrocatalytic activity of the electrodes prepared from polyhedral and spherical Ni NCs was initially investigated by $\mathrm{CV}$ within the potential range $0-1.0 \mathrm{~V} v s$. $\mathrm{Hg} / \mathrm{HgO}$, in the presence and absence of $0.2 \mathrm{M}$ methanol in a $1.0 \mathrm{M} \mathrm{KOH}$ solution (Figure 2). The current density of polyhedral NC-based electrodes was systematically and significantly higher than those prepared from spherical NCs.
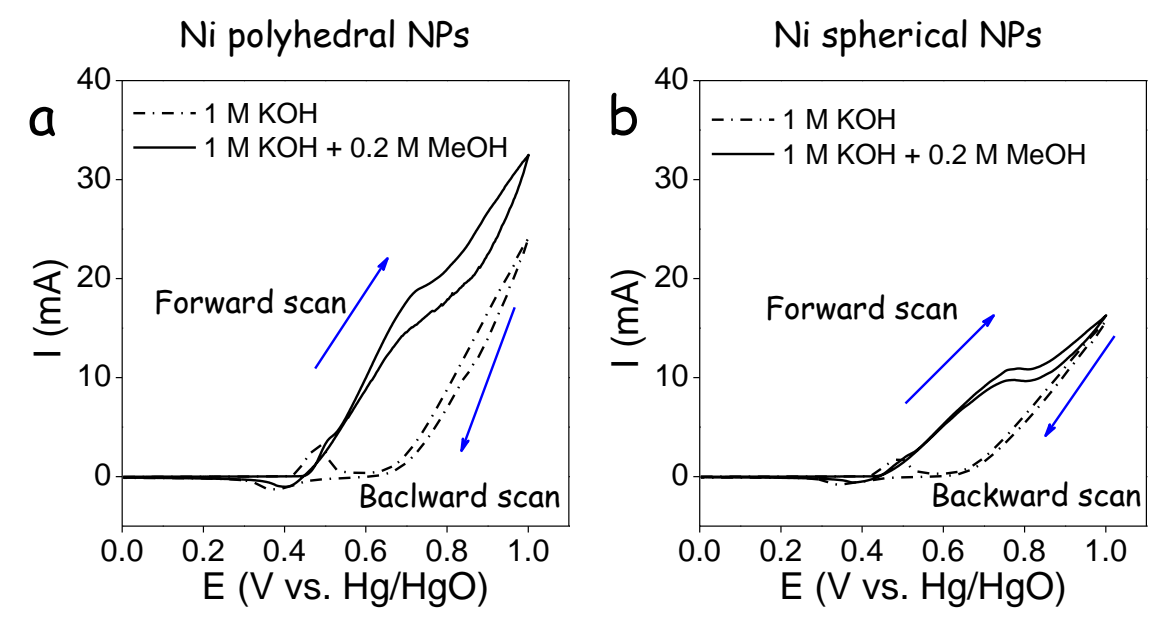

Figure 2. Cyclic voltammograms of Ni electrodes in $1.0 \mathrm{M} \mathrm{KOH}$ solution in the absence and presences of 0.2 $\mathrm{M}$ methanol at a scan rate of $50 \mathrm{mV} \mathrm{s}^{-1}$ in 0-1.0 V: (a) Polyhedral NCs (b) spherical NCs. 
In alkaline media, $\mathrm{Ni}$ is oxidized to $\mathrm{Ni}(\mathrm{OH})_{2}$. When increasing the applied voltage, an anodic peak at ca. 0.43 $\mathrm{V}$ vs. $\mathrm{Hg} / \mathrm{HgO}$ is ascribed to the oxidation of $\mathrm{Ni}(\mathrm{OH})_{2}$ to $\mathrm{NiOOH}^{6}{ }^{6}$ In the absence of methanol, at higher potential values, $>0.75 \mathrm{~V}$ vs. $\mathrm{Hg} / \mathrm{HgO}$, a rise in current density associated with the oxygen evolution from water (OER) is clearly observed. ${ }^{27}$ In the reverse scan, $\mathrm{NiOOH}$ is reduced back to $\mathrm{Ni}(\mathrm{OH})_{2}$ at ca. $0.35 \mathrm{~V} v s$. $\mathrm{Hg} / \mathrm{HgO}$.

In the presence of methanol, the oxidation of $\mathrm{Ni}(\mathrm{OH})_{2}$ to $\mathrm{NiOOH}$ triggers the electrocatalytic oxidation of methanol, fusing the increase of current density of the two chemical reactions together:

$\mathrm{Ni}(\mathrm{OH})_{2}+\mathrm{OH}^{-} \rightarrow \mathrm{NiOOH}+\mathrm{H}_{2} \mathrm{O}+\mathrm{e}^{-}$

$\mathrm{NiOOH}+\mathrm{CH}_{3} \mathrm{OH} \rightarrow \mathrm{Ni}(\mathrm{OH})_{2}+$ products

being the possible products/intermediates of the methanol electrooxidation mainly formic acid, carbonate, formaldehyde, $\mathrm{CO}$ and $\mathrm{CO}_{2}$. At higher applied voltages, the large increase of current corresponding to the OER adds on.

We further investigated the kinetics of the $\mathrm{Ni}(\mathrm{OH})_{2}$ oxidation to $\mathrm{NiOOH}$ as a function of the $\mathrm{KOH}$ concentration for the polyhedral Ni NCs by CV (Figure 4 and Table 2). We observed the anodic peak current $\left(I_{p a}\right)$ to significantly increase and shift to lower potentials $\left(E_{p a}\right)$ with the $\mathrm{KOH}$ concentration, while the cathodic peak current $\left(I_{p c}\right)$ just moderately raised at a similar potential $\left(E_{p c}\right)$. Accordingly, the potential difference between the two redox peaks $(\Delta E)$ was strongly reduced when increasing the $\mathrm{KOH}$ concentration, indicating enhanced electron transfer kinetics between the electrode surface and the electrolyte. The linear fit of the $\log j$ vs $\log \left[\mathrm{OH}^{-}\right]$plot gave a $\mathrm{OH}^{-}$reaction order of 1.2 for the $\mathrm{Ni}(\mathrm{OH})_{2}$ to $\mathrm{NiOOH}$ reaction, which was consistent with previous literature reports displaying a reaction order of 1 .

When increasing the sweep rate, the position of the anodic peak shifted to higher potential values and the cathodic peak moved to lower potential values (Figure 3 and S6). The current densities of both anodic and cathodic peaks rose with increasing scan rate. In the low scan rate range $\left(v<80 \mathrm{mV} \mathrm{s}^{-1}\right)$, both peak currents were linearly proportional to the scan rate. From the average slope of the anodic and cathodic peaks $v s . v$, the surface coverage of redox species $\left(\Gamma^{*}\right)$ was estimated (Table 1$):^{28}$

$$
I_{p}=\left(\frac{n^{2} F^{2}}{4 R T}\right) A \Gamma^{*} v
$$

where $\mathrm{n}, \mathrm{F}, \mathrm{R}, \mathrm{T}$ and $\mathrm{A}$ are the number of transferred electrons (assumed to be 1), the Faraday constant (96845 $\left.\mathrm{C} \mathrm{mol}^{-1}\right)$, the gas constant $\left(8.314 \mathrm{~J} \mathrm{~K}^{-1} \mathrm{~mol}^{-1}\right)$, temperature and the geometric surface area of the glassy carbon electrodes $\left(0.196 \mathrm{~cm}^{2}\right)$, respectively. From this equation, the surface coverage of redox species was found to slightly increase with the $\mathrm{KOH}$ concentration, from $5.7 \times 10^{-8} \mathrm{~mol} \mathrm{~cm}^{-2}$ in $0.1 \mathrm{M} \mathrm{KOH}$ to 1.93 $\times 10^{-7} \mathrm{~mol} \mathrm{~cm}^{-2}$ in $1.0 \mathrm{M} \mathrm{KOH}$. 
In the high scan rate range $\left(80-150 \mathrm{mV} \mathrm{s}^{-1}\right)$, a linear relationship could be fitted to the dependence of the peak current density with the square root of the voltage scan rate. This dependence is generally related with a diffusion-limited $\mathrm{Ni}(\mathrm{OH})_{2} \leftrightarrow \mathrm{NiOOH}$ redox reaction, where the proton diffusion within the particle is generally considered the diffusion process that limits the reaction rate: ${ }^{28}$

$$
I_{p}=2.69 \times 10^{5} n^{3 / 2} A D^{1 / 2} C v^{1 / 2}
$$

where $C$ is the initial concentration of redox species. In the case of ca. $16 \mathrm{~nm} \mathrm{NCs,} \mathrm{the} \mathrm{related} \mathrm{proton}$ diffusion lengths are comparatively very small. Using the above equation, which is valid for a semi-infinite layer, rate limiting diffusion coefficients at different $\mathrm{KOH}$ concentrations were estimated at $1.19 \times 10^{-10}, 0.45$ $\times 10^{-10}, 0.81 \times 10^{-10}, 1.20 \times 10^{-10}$ and $2.02 \times 10^{-10} \mathrm{~cm}^{2} \mathrm{~s}^{-1}$ in $0.1,0.3,0.5,0.7$ and $1.0 \mathrm{M} \mathrm{KOH}$ solution, respectively. When calculating these parameters for spherical NCs (Figure S7), we realized that the coverage $\Gamma^{*}$ in spherical Ni NPs was much lower than in polyhedral NCs, $3.19 \times 10^{-8} \mathrm{~mol} \mathrm{~cm}^{-2}$ in $1.0 \mathrm{M} \mathrm{KOH}$. On the other hand, the rate limiting diffusion coefficients was higher, $1.45 \times 10^{-9} \mathrm{~cm}^{2} \mathrm{~s}^{-1}$ in $1.0 \mathrm{M} \mathrm{KOH}$.

The electrochemically active surface area (ECSA) was estimated by using the electrochemical double-layer capacitance $\left(\mathrm{C}_{\mathrm{dl}}\right)$ on the basis of $\mathrm{CVs}$ recorded at different scan rates in the non-faradaic potential range 0-0.1 $\mathrm{V}$ vs. $\mathrm{Hg} / \mathrm{HgO}$ (Figure 3.e) ${ }^{29}$ Plotting the capacitive current $\left(I_{c}\right)$ vs. the scan rate $(v)$ yielded a straight line with a slope equal to $\mathrm{C}_{\mathrm{dl}}$ (Figure $3 \mathrm{f}$ ) and then ECSA was calculated by dividing $\mathrm{C}_{\mathrm{dl}}$ by the specific capacitance $\left(\mathrm{C}_{\mathrm{s}}\right):^{30}$

$$
E C S A=C_{d l} / C_{S}
$$

where $\mathrm{C}_{\mathrm{s}}$ is a general specific capacitance of $0.04 \mathrm{mF} \mathrm{cm} \mathrm{c}^{-2}$ based on typical value reported for metal electrodes in aqueous $\mathrm{NaOH}$ solution. ${ }^{31}$ For polyhedral $\mathrm{NC}$ electrodes in $1.0 \mathrm{M} \mathrm{KOH}$ solution, the calculated value of $\mathrm{C}_{\mathrm{dl}}$ was $0.25 \mathrm{mF} \mathrm{cm}^{-2}$, and the ECSA was $6.5 \mathrm{~cm}^{-2}$. 

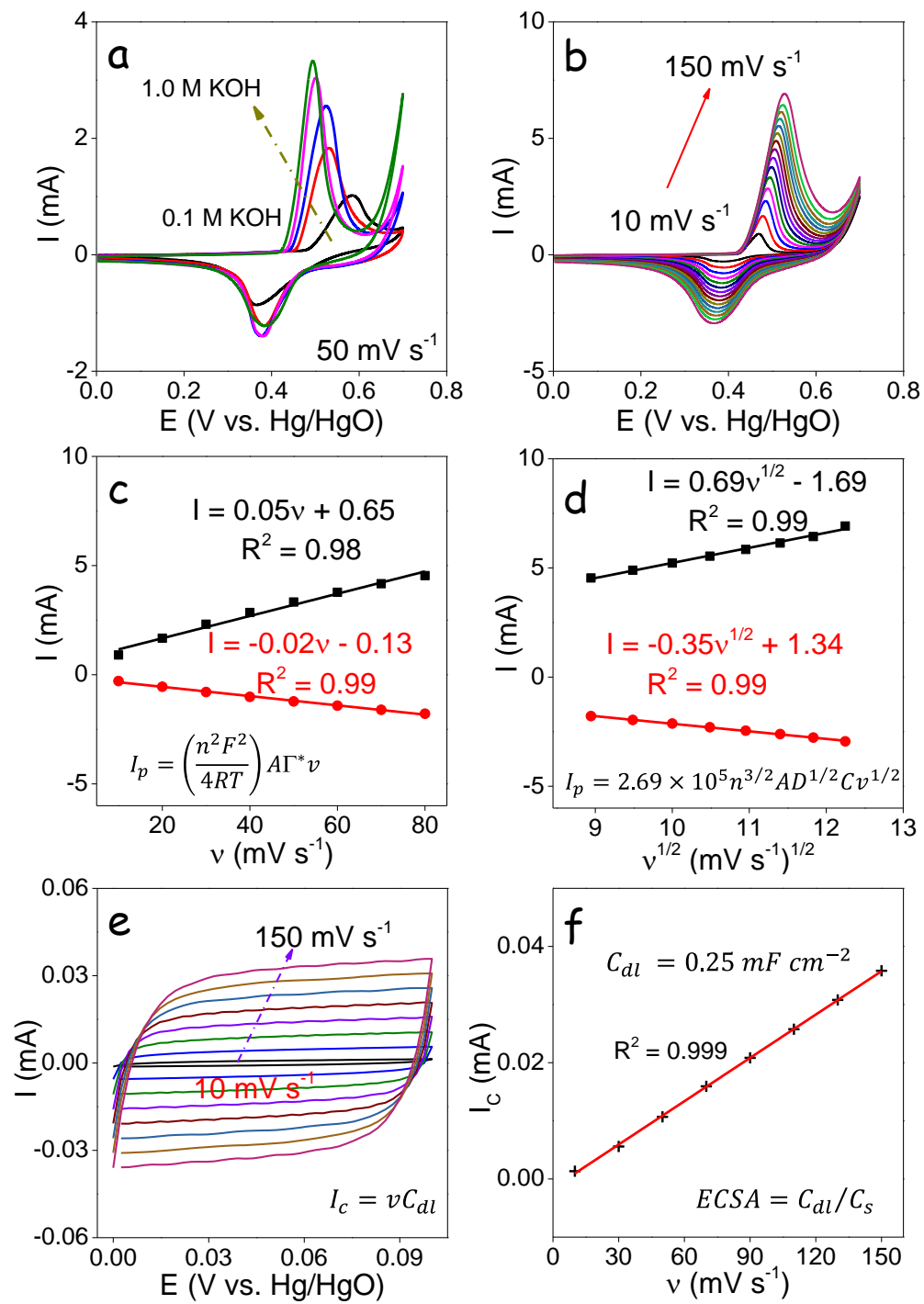

Figure 3. (a) CV of polyhedral $\mathrm{Ni} N C s$ in $0.1 \mathrm{M}, 0.3 \mathrm{M}, 0.5 \mathrm{M}, 0.7 \mathrm{M}$ and $1.0 \mathrm{M} \mathrm{KOH}$ solution at a scan rate of $50 \mathrm{mV} \mathrm{s}^{-1}$. (b) $\mathrm{CV}$ of polyhedral Ni NCs in $1.0 \mathrm{M} \mathrm{KOH}$ solution at increasingly higher potentials sweep rates: $10,20,30,40,50,60,70,80,90,100,110,120,130,140,150 \mathrm{mV} \mathrm{s}^{-1}$. (c) Linear fitting of anodic and cathodic peak current densities with the scan rate in the low scan rate range $\left(10-80 \mathrm{mV} \mathrm{s}^{-1}\right)$ for polyhedral $\mathrm{Ni}$ NCs. (d) Linear fitting of anodic and cathodic peak current densities to the square roots of the scan rates in the high scan rate range $\left(80-150 \mathrm{mV} \mathrm{s}^{-1}\right)$ for polyhedral Ni NCs. (e) CVs in the double layer region for polyhedral NCs electrodes at scan rates of $10,30,50,70,90,110,130,150 \mathrm{mV} \mathrm{s}^{-1}$ in the non-faradaic range of $0-0.1 \mathrm{~V}$ vs. $\mathrm{Hg} / \mathrm{HgO}$. (f) Corresponding linear fit of the capacitive current vs. scan rates to calculate $\mathrm{C}_{\mathrm{dl}}$ and the ECSA.

Table 1. Summary of the electrocatalytic performance of Ni polyhedral NCs based electrodes in variable concentration of $\mathrm{KOH}$.

\begin{tabular}{|c|c|c|c|c|c|c|c|}
\hline $\begin{array}{l}C_{K O H} \\
\text { (M) }\end{array}$ & $\begin{array}{c}\boldsymbol{I}_{p a} \\
(\mathbf{m A})\end{array}$ & $\begin{array}{c}E_{p a} \\
(\mathrm{~V} v s . \mathrm{Hg} / \mathrm{HgO})\end{array}$ & $\begin{array}{c}\boldsymbol{I}_{p c} \\
(\mathbf{m A})\end{array}$ & $\begin{array}{c}E_{p c} \\
(\mathbf{V} v s . \mathbf{H g} / \mathbf{H g O})\end{array}$ & $\begin{array}{l}\Delta E \\
(\mathbf{V})\end{array}$ & 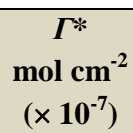 & $\begin{array}{c}D \\
\mathrm{~cm}^{2} \mathrm{~s}^{-1} \\
\left(\times 10^{-9}\right)\end{array}$ \\
\hline 0.1 & 1.021 & 0.583 & -0.862 & 0.366 & 0.217 & 0.57 & 0.12 \\
\hline
\end{tabular}




\begin{tabular}{|l|l|l|l|l|l|l|l|}
\hline 0.3 & 1.833 & 0.532 & -1.225 & 0.383 & 0.149 & 1.09 & 0.45 \\
\hline 0.5 & 2.556 & 0.525 & -1.395 & 0.378 & 0.146 & 1.47 & 0.81 \\
\hline 0.7 & 3.040 & 0.500 & -1.400 & 0.381 & 0.120 & 1.71 & 1.20 \\
\hline 1.0 & 3.331 & 0.493 & -1.222 & 0.383 & 0.110 & 1.93 & 2.02 \\
\hline
\end{tabular}

$\mathrm{I}_{\mathrm{pa}}, \mathrm{E}_{\mathrm{pa}}, \mathrm{I}_{\mathrm{pc}}, \mathrm{E}_{\mathrm{pc}}$ and $\Delta \mathrm{E}$ were measured from the CVs in solution at a scan rate of $50 \mathrm{mV} \mathrm{s}^{-1}$ in $0.1 \mathrm{M}, 0.3 \mathrm{M}$, $0.5 \mathrm{M}, 0.7 \mathrm{M}$ and $1.0 \mathrm{M} \mathrm{KOH}$ solution.

Figures $4 \mathrm{a}$ and S8 display CV $\left(50 \mathrm{mV} \mathrm{s}^{-1}\right)$ curves of the Ni polyhedral NCs electrocatalyts in a $1.0 \mathrm{M} \mathrm{KOH}$ media containing different methanol concentrations, from $0.1 \mathrm{M}$ to $1.0 \mathrm{M}$. In addition, the electrochemical response towards methanol concentration for spherical Ni NPs in $1.0 \mathrm{M} \mathrm{KOH}$ containing $1.0 \mathrm{M}$ methanol is displayed in Figure S9. It can be observed that the $\mathrm{Ni}(\mathrm{OH})_{2}$ oxidation peak gradually disappeared within the large current density increase related to the MOR when the methanol concentration increased. Figure $4 \mathrm{~b}$ summarizes the current density $(0.7 \mathrm{~V}$ vs. $\mathrm{Hg} / \mathrm{HgO})$ as a function of methanol concentration in 0.1-1.0 M $\mathrm{KOH}$ media. Higher current densities were obtained in the presence of higher concentration of $\mathrm{KOH}$. At the same time, the current density rose gradually with the methanol concentration until $0.3 \mathrm{M}$ of methanol was reached, followed by a rather lazy variation. Specifically, current densities around $110 \mathrm{~mA} \mathrm{~cm}^{-2}$ were measured in 1.0 M KOH solution containing 0.5 M methanol. As shown in Figure S10, a linear fit of the logarithmic plot of the current density of MOR versus the methanol concentration for the Ni polyhedral NCs based electrode pointed toward an apparent methanol reaction order of around 0.3 at $0.6 \mathrm{~V}$.

Figure $4 \mathrm{c}$ displays the CVs of the polyhedral NCs based electrode in $0.1 \mathrm{M}, 0.3 \mathrm{M}, 0.5 \mathrm{M}, 0.7 \mathrm{M}$ and $1.0 \mathrm{M}$ $\mathrm{KOH}(\mathrm{CB}$ electrode in $1.0 \mathrm{M} \mathrm{KOH})$ containing $1.0 \mathrm{M}$ methanol in the potential window $0-0.7 \mathrm{~V}$. The current density in the vertical axis was normalized by the surface area of the electrode. The results show that the MOR performance is highly related to the alkaline media concentration. At equal $\mathrm{KOH}$ and methanol concentrations, polyhedral NCs provided close to a twofold higher current density.

The mass current density obtained in the presence of variable $\mathrm{KOH}$ concentrations and 1.0 M methanol from Ni polyhedral NCs based electrodes is listed in Table S1, where it is compared with literature results. At 1.0 $\mathrm{M} \mathrm{KOH}$, the mass current density was $2016.2 \mathrm{~mA} \mathrm{mg}^{-1}$ at $0.6 \mathrm{~V}$ and $3162 \mathrm{~mA} \mathrm{mg}^{-1}$ and $0.7 \mathrm{~V}$ for the $\mathrm{Ni}$ polyhedral NCs electrodes, which is a twofold higher than that obtained for spherical NCs and well above values previously reported in literature. 

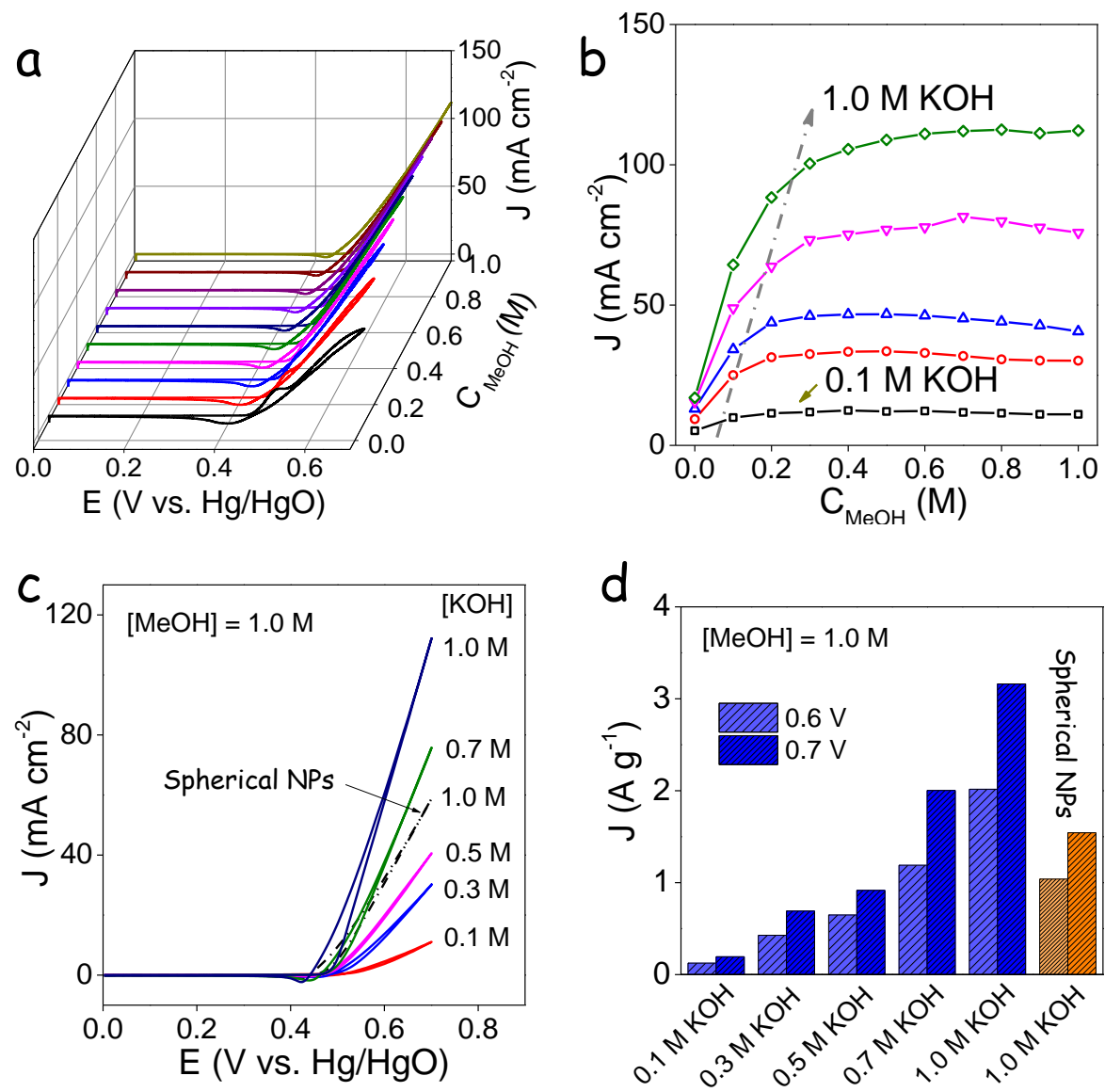

Figure 4. (a) CVs of an electrode based on polyhedral Ni NCs in $1.0 \mathrm{M} \mathrm{KOH}$ solution with different methanol concentrations, from $0.1 \mathrm{M}$ to $1.0 \mathrm{M}$, at a scan rate of $50 \mathrm{mV} \mathrm{s}^{-1}$. (b) Comparison of the current density at $0.7 \mathrm{~V}$ of an electrode based on $\mathrm{Ni}$ polyhedral $\mathrm{NCs}$ as a function of methanol concentration, from 0.1 M to 1.0 M. (c) CVs of an electrode based on Ni polyhedral NCs in $0.1 \mathrm{M}, 0.3 \mathrm{M}, 0.5 \mathrm{M}, 0.7 \mathrm{M}$ and 1.0 $\mathrm{M} \mathrm{KOH}$ containing 1.0 M methanol at a scan rate of $50 \mathrm{mV} \mathrm{s}^{-1}$. Data for an electrode based on Ni spherical NPs measured in $1.0 \mathrm{M} \mathrm{KOH}$ with $1.0 \mathrm{M}$ methanol is also plotted for comparison. (d) The mass current density of an electrode based on Ni polyhedral NCs in $0.1 \mathrm{M}, 0.3 \mathrm{M}, 0.5 \mathrm{M}, 0.7 \mathrm{M}, 1.0 \mathrm{M} \mathrm{KOH}$ and of an electrode based on Ni spherical NCs in $1.0 \mathrm{M} \mathrm{KOH}$, both in $1.0 \mathrm{M}$ methanol at $0.6 \mathrm{~V}$ and $0.7 \mathrm{~V}$. Note: the mass current density at $0.7 \mathrm{~V}$ was corrected by subtracting the OER contribution (obtained from measurements in the absence of methanol) to the current densities measured in the presence of $\mathrm{KOH}$ and methanol.

The long-term activities of the as-prepared electrodes based on $\mathrm{Ni} \mathrm{NCs}$ were evaluated by chronoamperometry (CA) in $1.0 \mathrm{M} \mathrm{KOH}$ solution containing $1.0 \mathrm{M}$ Methanol at $0.6 \mathrm{~V}$ vs. $\mathrm{Hg} / \mathrm{HgO}$ for 35000s. Figure 5a shows data for an electrode based on Ni polyhedral NCs. The current density of such electrodes suffered a relatively important decrease during the first few hours of operation $(30 \%$ loss after 10000s), which was additionally measured by CV (Figure 5b). After this initial lost, the current density quasi-stabilized at a value of ca. $37 \mathrm{~mA} \mathrm{~cm}^{-2}$. During the last hours of operation tested, from $\mathrm{t}=20000$ to 
$\mathrm{t}=30000$, a current density decrease of just a $0.5 \%$ was obtained. Notice that the mass current density at which the electrode stabilized, $\sim 1200 \mathrm{~mA} \mathrm{mg}^{-1}$, was still significantly higher than the maximum current densities published for Ni-based electrodes in previous reports. The initial current density decay is probably ascribed to the blocking of active sites by reaction products that are moderately strongly bind to the $\mathrm{Ni}$ surface, after some hours of operation, the coverage of these species may stabilize either because of having reached an equilibrium adsorption/desorption or because of the complete blocking of certain preferential sites. In any case, the density of surface reaction sites apparently decreases in around a $35 \%$ before the catalyst stabilizes.

Electrodes based on Ni polyhedral NCs displayed improved stabilities over those based on Ni spherical NCs which suffered a $58 \%$ loss after 10000 s in $1.0 \mathrm{M} \mathrm{KOH}$ with $1.0 \mathrm{M}$ methanol at $0.6 \mathrm{~V}$ vs. $\mathrm{Hg} / \mathrm{HgO}$ (Figure S11). This experimental fact should be explained by the different surface sites of polyhedral and spherical NCs, but additional work is required to exactly asses the mechanism of improvement of the electrode stability.
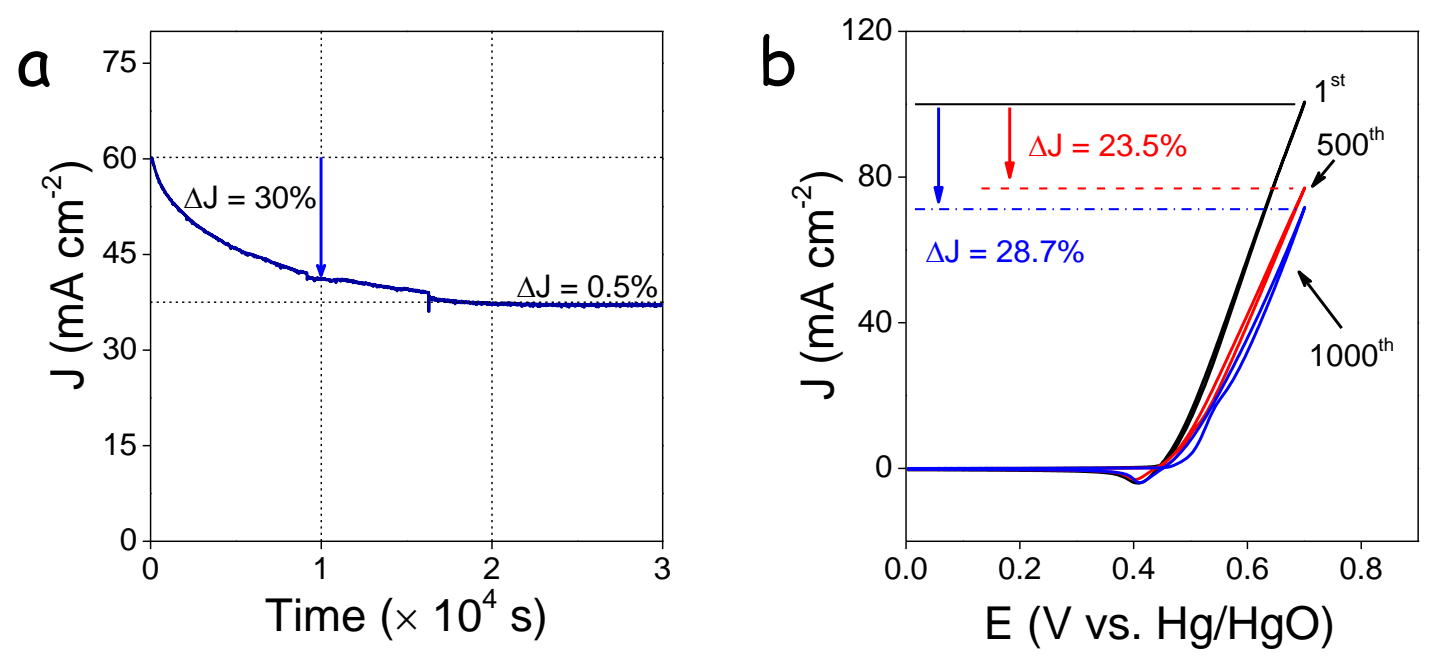

Figure 5. (a) CA response of Ni polyhedral NCs based electrodes in $1.0 \mathrm{M} \mathrm{KOH}$ and $1.0 \mathrm{M}$ methanol at 0.6 V for $10000 \mathrm{~s}$. (b) CVs of the Ni polyhedral NCs based electrode in $1.0 \mathrm{M} \mathrm{KOH}$ electrolyte with $1.0 \mathrm{M}$ methanol at a scan rate of $100 \mathrm{mV} \mathrm{s}^{-1}$ in the potential range $0-0.7 \mathrm{~V}$ at cycles $1^{\text {st }}, 500^{\text {th }}, 1000^{\text {th }}$.

\section{Conclusion}

Colloidal Ni polyhedral NCs $(16 \pm 2 \mathrm{~nm})$ with predominant (110) facets were synthesized and tested as electrodes for methanol electrooxidation. Results obtained from electrodes based on Ni polyhedral NCs were compared with those obtained for electrodes based on Ni spherical NCs. Electrocatalytic properties were first investigated in variable concentrations of $\mathrm{KOH}$. We found the $\mathrm{Ni}(\mathrm{OH})_{2} \leftrightarrow \mathrm{NiOOH}$ redox reaction rate was first order with the $\mathrm{KOH}$ concentration. The surface coverage of active species was much higher in electrodes based on polyhedral Ni NCs than in spherical ones. On the other hand, the rate limiting diffusion 
coefficient was higher in electrodes based on spherical NCs. Electrodes based on Ni polyhedral NCs displayed impressive current densities $\left(59.4 \mathrm{~mA} \mathrm{~cm}^{-2}\right)$ and mass activities $\left(2016 \mathrm{~mA} \mathrm{mg}^{-1}\right)$ at $0.6 \mathrm{~V} v s$. $\mathrm{Hg} / \mathrm{HgO}$ in the presence of $1.0 \mathrm{M}$ methanol and $1.0 \mathrm{M} \mathrm{KOH}$, which corresponds to a twofold increase over electrodes based on spherical Ni NCs and over most previous Ni-baed electrocatalyts previously reported. Such superior catalytic performance should be ascribed to the proper facets of Ni polyhedral NCs, which provided abundant active sites to promote the oxidation of methanol in alkaline media. Electrodes based on faceted polyhedral NCs displayed a 30\% loss of activity during the first few operation hours, but activity stabilized to around a $65 \%$ of the initial value after ca. 20000 s operation. These results suggest $\mathrm{Ni}$ polyhedral NCs with predominant (110) facets to be among the best candidates for MOR.

\section{Supporting Information}

Supplementary material related to this article can be found.

\section{Author Information}

Corresponding Author

* Andreu Cabot: acabot@irec.cat.

*Zhishan luo: luozs@mail.sustc.edu.cn

\section{Author Contributions}

The manuscript was prepared through the contribution of all authors. A. Cabot and Z. Luo conceived and guided the project, and supervised the work. J. Li designed the experiments, produced the NCs, conducted XRD, TEM and FT-IR characterization, electrochemical measurements, and wrote the first draft of the manuscript. Y. Zuo, J. Liu, X. Yu and R. Du significantly contributed to the results discussion. T. Zhang, M. F. Infante-Carrió, P. Tang and J. Arbiol performed structural and compositional NCs characterization by means of HRTEM and EELS, and discussed the results. J. Llorca measured and discussed XPS data. The manuscript was corrected and improved by all authors.

\section{Acknowledgments}

This work was supported by the European Regional Development Funds and by the Spanish Ministerio de Economía y Competitividad through the project SEHTOP (ENE2016-77798-C4-3-R) and VALPEC (ENE2017-85087-C3). J. Li and T. Zhang thank the China Scholarship Council (CSC) for scholarship support. T. Zhang and J. Arbiol acknowledge funding from Generalitat de Catalunya 2017 SGR 327. ICN2 acknowledges support from the Severo Ochoa Programme (MINECO, Grant no. SEV-2013-0295). IREC and ICN2 are funded by the CERCA Programme / Generalitat de Catalunya. Part of the present work has been performed in the framework of Universitat Autònoma de Barcelona Materials Science PhD program. J. 
Llorca is a Serra Húnter Fellow and is grateful to ICREA Academia program and to MINECO/FEDER grant ENE2015-63969-R and funding from Generalitat de Catalunya 2017 SGR 128.

\section{Conflict of interest}

The authors declare no competing financial interest.

\section{References}

$1 \quad$ R. G. Minet, Science (80-. )., 1967, 157, 1373.

2 X. Zhao, M. Yin, L. Ma, L. Liang, C. Liu, J. Liao, T. Lu and W. Xing, Energy Environ. Sci., 2011, 4, 2736.

3 J. N. Tiwari, R. N. Tiwari, G. Singh and K. S. Kim, Nano Energy, 2013, 2, 553-578.

4 N. Kakati, J. Maiti, S. H. Lee, S. H. Jee, B. Viswanathan and Y. S. Yoon, Chem. Rev., 2014, 114, 12397-12429.

5 A. Serov and C. Kwak, Appl. Catal. B Environ., 2009, 90, 313-320.

6 X. Cui, W. Guo, M. Zhou, Y. Yang, Y. Li, P. Xiao, Y. Zhang and X. Zhang, ACS Appl. Mater. Interfaces, 2015, 7, 493-503.

7 R. M. Abdel Hameed and R. M. El-Sherif, Appl. Catal. B Environ., 2015, 162, 217-226.

8 H. Sun, Y. Ye, J. Liu, Z. Tian, Y. Cai, P. Li and C. Liang, Chem. Commun., 2018, 54, 1563-1566.

9 G. S. Ferdowsi, S. A. Seyedsadjadi and A. Ghaffarinejad, J. Nanostructure Chem., 2015, 5, 17-23.

10 J. Li, Z. Luo, Y. Zuo, J. Liu, T. Zhang, P. Tang, J. Arbiol, J. Llorca and A. Cabot, Appl. Catal. B Environ., 2018, 234, 10-18.

11 J. Li, Z. Luo, F. He, Y. Zuo, C. Zhang, J. Liu, X. Yu, R. Du, T. Zhang, M. F. Infante-Carrió, P. Tang, J. Arbiol, J. Llorca and A. Cabot, J. Mater. Chem. A, 2018, 6, 22915-22924.

12 N. A. M. Barakat, M. Motlak, B. S. Kim, A. G. El-Deen, S. S. Al-Deyab and A. M. Hamza, J. Mol. Catal. A Chem., 2014, 394, 177-187.

13 Y. Yu, Q. Yang, X. Li, M. Guo and J. Hu, Green Chem., 2016, 18, 2827-2833.

14 A. Roy, H. S. Jadhav, G. M. Thorat and J. G. Seo, New J. Chem., 2017, 41, 9546-9553.

15 S.-L. Yau, F.-R. F. Fan, T. P. Moffat and A. J. Bard, J. Phys. Chem., 1994, 98, 5493-5499.

16 Z. Luo, J. Lu, C. Flox, R. Nafria, A. Genç, J. Arbiol, J. Llorca, M. Ibáñez, J. R. Morante and A. Cabot, J. Mater. Chem. A, 2016, 4, 16706-16713.

17 G. You, J. Jiang, M. Li, L. Li, D. Tang, J. Zhang, X. C. Zeng and R. He, ACS Catal., 2018, 8, 132143.

18 H. Kita, Y. Gao, S. Ye and K. Shimazu, Bull. Chem. Soc. Jpn., 2006, 66, 2877-2882.

19 J. C. Davies, B. E. Hayden and D. J. Pegg, Surf. Sci., 2000, 467, 118-130.

20 J. Clavilier, A. Rodes, K. El Achi and M. Zamakhchari, J. Chim. Phys., 2017, 88, 1291-1337. 
21 C. F. Mai, C. H. Shue, Y. C. Yang, L. Y. O. Yang, S. L. Yau and K. Itaya, Langmuir, 2005, 21, 4964-4970.

22 Y. Chen, X. Luo, H. She, G.-H. Yue and D.-L. Peng, J. Nanosci. Nanotechnol., 2009, 9, 5157-5163.

23 A. T. Fafarman, W. K. Koh, B. T. Diroll, D. K. Kim, D. K. Ko, S. J. Oh, X. Ye, V. Doan-Nguyen, M. R. Crump, D. C. Reifsnyder, C. B. Murray and C. R. Kagan, J. Am. Chem. Soc., 2011, 133, $15753-$ 15761.

24 J. Li, X. Xu, Z. Luo, C. Zhang, Y. Zuo, T. Zhang, P. Tang, M. F. Infante-Carrió, J. Arbiol, J. Llorca, J. Liu and A. Cabot, ChemSusChem, 2019, 12, 1451-1458.

25 J. Li, X. Xu, Z. Luo, C. Zhang, X. Yu, Y. Zuo, T. Zhang, P. Tang, J. Arbiol, J. Llorca, J. Liu and A. Cabot, Electrochim. Acta, 2019, 304, 246-254.

26 J. Liu, Z. Luo, J. Li, X. Yu, J. Llorca, D. Nasiou, J. Arbiol, M. Meyns and A. Cabot, Appl. Catal. B Environ., 2019, 242, 258-266.

27 D. Wu, W. Zhang and D. Cheng, ACS Appl. Mater. Interfaces, 2017, 9, 19843-19851.

28 A. J. Bard and L. R. Faulkner, Anti-Corrosion Methods Mater., 2001, 50, 1-850.

29 J. D. Benck, Z. Chen, L. Y. Kuritzky, A. J. Forman and T. F. Jaramillo, ACS Catal., 2012, 2, 19161923.

30 C. C. L. McCrory, S. Jung, I. M. Ferrer, S. M. Chatman, J. C. Peters and T. F. Jaramillo, J. Am. Chem. Soc., 2015, 137, 4347-4357.

31 C. C. L. McCrory, S. Jung, J. C. Peters and T. F. Jaramillo, J. Am. Chem. Soc., 2013, 135, 1697716987. 